\title{
Low-Loss Superconducting Nanowire Circuits Using a Neon Focused Ion Beam
}

\author{
J. Burnett, ${ }^{*}$ J. Sagar, O. W. Kennedy, P. A. Warburton, and J. C. Fenton ${ }^{\dagger}$ \\ London Centre for Nanotechnology, UCL, 17-19 Gordon Street, London WC1H OAH, United Kingdom
}

(Received 8 March 2017; revised manuscript received 30 May 2017; published 31 July 2017)

\begin{abstract}
We present low-temperature measurements of low-loss superconducting nanowire-embedded resonators in the low-power limit relevant for quantum circuits. The superconducting resonators are embedded with superconducting nanowires with widths down to $20 \mathrm{~nm}$ using a neon focused ion beam. In the low-power limit, we demonstrate an internal quality factor up to $3.9 \times 10^{5}$ at $300 \mathrm{mK}$ [implying a two-level-systemlimited quality factor up to $2 \times 10^{5}$ at $10 \mathrm{mK}$ ], not only significantly higher than in similar devices but also matching the state of the art of conventional Josephson-junction-embedded resonators. We also show a high sensitivity of the nanowire to stray infrared photons, which is controllable by suitable precautions to minimize stray photons in the sample environment. Our results suggest that there are excellent prospects for superconducting-nanowire-based quantum circuits.
\end{abstract}

DOI: 10.1103/PhysRevApplied.8.014039

\section{INTRODUCTION}

Quantum circuits based on conventional Josephson junctions have begun to tackle real-world problems [1]. This has been despite high decoherence produced by the loss $[2,3]$ and noise [4,5] caused by parasitic two-level systems (TLSs) [6,7]. In principle, superconducting nanowires can provide a route to low-decoherence quantum circuits due to their monolithic structure and lack of a TLS-hosting oxide layer. To date, superconducting nanowires with cross-sectional areas approaching the coherence length have demonstrated a variety of Josephson [8,9] and phase-slip [10-13] effects, but features such as their unconventional current-phase relationships [14] remain unexploited in quantum circuits. Previous demonstrations of superconducting nanowire-embedded resonators exhibit unusually high dissipation, with internal quality factors $\left(Q_{i}\right)$ below $5 \times 10^{3}[10-12,15]$, far lower than in similar conventional Josephson-junction-based circuits [16,17]. In general, the performance of nanowire-embedded resonators can be limited by material quality, interface imperfections, resist residues, and the measurement environment.

We demonstrate superconducting nanowire-embedded circuits with single photon $Q_{i}$ up to $3.9 \times 10^{5}$, comparable to or even better than conventional Josephson-junction resonators. Superconducting nanowires with widths down to $20 \mathrm{~nm}$ are fabricated with a neon focused ion beam (FIB). We study the loss in our devices within the well-established

\footnotetext{
*burnett@chalmers.se

j.fenton@ucl.ac.uk
}

Published by the American Physical Society under the terms of the Creative Commons Attribution 4.0 International license. Further distribution of this work must maintain attribution to the author(s) and the published article's title, journal citation, and DOI. framework of loss mechanisms in superconducting resonators $[2,3,7,18,19]$ to determine which factors are significant in limiting their performance. The vastly improved $Q_{i}$ demonstrates that the detrimental effects can be sufficiently reduced and shows that competitive quantum circuits can be based on monolithic nanowire technology.

\section{METHODS}

Superconducting 20-nm-thick NbN films are deposited on sapphire by dc magnetron sputtering from a $99.99 \%$ pure $\mathrm{Nb}$ target in a 1:1 Ar: $\mathrm{N}_{2}$ atmosphere. The vacuum chamber is pumped to $6 \times 10^{-7} \mathrm{mbar}$ before sputtering at a pressure of $3.5 \times 10^{-3} \mathrm{mbar}$ and power of $200 \mathrm{~W}$. The superconducting critical temperature $T_{c}$ is $10 \mathrm{~K}$, with a sheet resistance of $450 \Omega / \mathrm{sq}$. Electron-beam lithography (EBL) is used to pattern $\lambda / 4$ and $\lambda / 2$ coplanar microwave resonators capacitively coupled to a common microwave feed line [shown in Fig. 1(d)]. The width of the central conductor is $10 \mu \mathrm{m}$, and the gap is $5 \mu \mathrm{m}$. This pattern is transferred from a 300-nm-thick layer of polymethyl methacrylate into the film by a reactive ion etch using a 2:1 ratio of $\mathrm{SF}_{6}: \mathrm{Ar}$ at $30 \mathrm{~W}$ and 30 mbar.

A neon FIB is used to directly pattern [20] nanowires in the central conductor of the microwave resonators at the current antinode; see Fig. 1(b). With an acceleration voltage of $15 \mathrm{kV}$, the clearance dose for the $\mathrm{NbN}$ film is approximately $0.3 \mathrm{nC} / \mu \mathrm{m}^{2}$. We choose $15 \mathrm{kV}$ as a compromise between minimizing the spot size and minimizing the lateral milling of the nanowire [21], leading to a fewminute mill time per $\mu \mathrm{m}^{2}$ for an approximately 1-pA beam current. By prior patterning of a sub-200-nm-wide precursor wire in the same EBL step as the resonator [shown in Fig. 1(c)], we minimize the mill time and the total neon flux that the nanowire is subject to. Several devices are measured, and Table I shows important parameters 

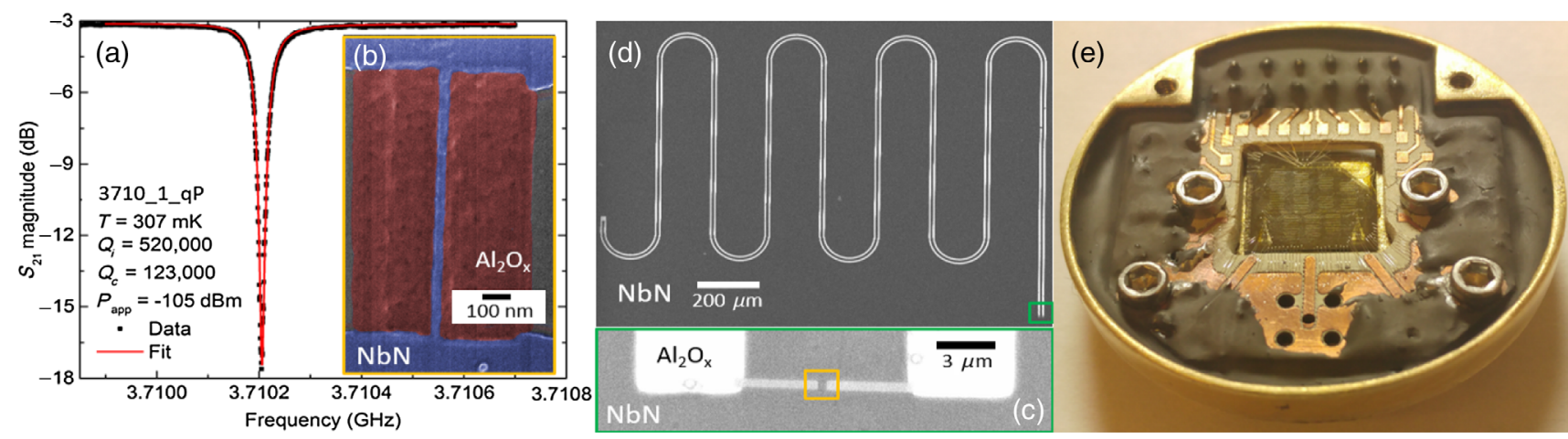

FIG. 1. (a) The $S_{21}$ magnitude response of the nanowire-embedded resonator 3710_1qP. The red line is a fit to Eq. (1). (b) A false-color He FIB micrograph of a neon FIB-milled nanowire (3710_1qP) with dimensions of $27 \mathrm{~nm}$ by $1.2 \mu \mathrm{m}$. The NbN is shown in blue, while the milled region is shown in red. (c),(d) Scanning electron micrographs of (c) the shorted end of a $\lambda / 4$ resonator before milling by neon FIB. (d) The whole $\lambda / 4$ resonator. (e) A photograph of the sample holder. In the center is a chip, which is wire bonded to a microwave printed circuit board; the dark material is ECCOSORB CR 117.

including the nanowire dimensions. The nanowire devices all feature two nanowires configured either in parallel so that the nanowires complete a superconducting loop [12] or in series with a wider segment in between [22]. Here, there is no external flux bias or external gate bias, so the nanowires are treated as simple constrictions within the superconductor.

The samples are enclosed within a brass box and cooled using a ${ }^{3} \mathrm{He}$ refrigerator containing a heavily attenuated microwave in line and out line with a cryogenic highelectron-mobility-transistor amplifier.

\section{RESULTS AND DISCUSSION}

Figure 1(a) shows the forward transmission $\left(S_{21}\right)$ magnitude response of a nanowire-embedded resonator at $307 \mathrm{mK}$ and for an applied microwave drive of $-105 \mathrm{dBm}$, demonstrating $Q_{i}=5.2 \times 10^{5}$. This $Q_{i}$ is significantly higher than in comparable nanowire-based devices [10-12,15]. This highlights the promise of the neon FIB and demonstrates that superconducting nanowires are not intrinsically lossy. The complex $S_{21}$ notch response of the superconducting resonators is fitted by [23]

$$
S_{21}(\nu)=a e^{j \theta} e^{-2 \pi j \nu \tau}\left[1-\frac{\left(Q_{L} /\left|Q_{c}\right|\right) e^{j \phi}}{1+2 j Q_{L}\left(\nu / \nu_{0}-1\right)}\right],
$$

where $\nu$ is the applied frequency, $\nu_{0}$ the resonance frequency, $Q_{L}$ the loaded quality factor, and $\left|Q_{c}\right|$ the absolute value of the coupling quality factor. $\phi$ accounts for impedance mismatches, $a$ describes a change in amplitude, $\theta$ describes a change in phase, and $\tau$ a change in the electronic delay. The internal quality factor $Q_{i}$ is defined by $1 / Q_{L}=1 / Q_{i}+$ $\operatorname{Re}\left(1 / Q_{c}\right)$, and the energy within the resonator is $W_{\text {sto }}=2 P_{\text {app }} S_{\text {min }} Q_{L} / \omega_{0}$, where $P_{\text {app }}$ is the applied microwave power (in watts), and $S_{\min }$ the normalized minimum of the resonator magnitude response. We describe the microwave power in terms of the average number of photons in the resonator $\langle n\rangle$ given by $\langle n\rangle=W_{\text {sto }} /\left(h \nu_{0}\right)$, where $h$ is Planck's constant.

To examine the effect of the neon FIB on the $\mathrm{NbN}$ film, we measure the resonator response as a function of the temperature (shown in Fig. 2). As the temperature decreases from 2 to $1 \mathrm{~K}$, the resonant frequency increases due to changes in the complex conductivity, which are described by $\left(\Delta \nu / \nu_{0}\right)=(\alpha / 2)\left(\Delta \sigma_{2} / \sigma_{2}\right)$, where $\left(\Delta \nu / \nu_{0}\right)$ is

TABLE I. Table of resonator parameters. Resonators are named by $\nu_{0}(\mathrm{MHz})$, their chip number $\lambda / 4$ (q) or $\lambda / 2(\mathrm{~h})$, and whether they are bare resonators (B), have nanowires in series (S), have nanowires in parallel (P), or are measured in an ECCOSORB-lined box (E). $\bar{w}$ refers to the nanowire widths. $\delta_{\mathrm{TLS}}^{i}$ comes from fits to Eq. (2), while $\delta_{\mathrm{TLS}}^{0}$ and $\delta_{\mathrm{QP}}$ come from fits to Eq. (3), and $F P_{\gamma} \chi$ come from fits to Eq. (4).

\begin{tabular}{lccccc}
\hline \hline Resonator & $\bar{w}(\mathrm{~nm})$ & $F \delta_{\mathrm{TLS}}^{i}\left(\times 10^{-6}\right)$ & $F P_{\gamma} \chi\left(\times 10^{-6}\right)$ & $F \delta_{\mathrm{TLS}}^{0}\left(\times 10^{-6}\right)$ & $\delta_{\mathrm{QP}}\left(\times 10^{-7}\right)$ \\
\hline 4094_1qB & $\cdots$ & 6 & 0.57 & 6.6 & 5.6 \\
3995_1hB & $\cdots$ & 6.3 & 0.61 & 6.2 & 6.9 \\
3675_2qBE & $\cdots$ & 9.8 & 1.11 & 9.5 & 5.6 \\
2739_2hBE & $\cdots$ & 12.6 & 1.21 & 10.2 & 13.3 \\
3710_1qP & 27,30 & 4.8 & 0.47 & 5.9 & 12.4 \\
3012_1hS & 47,48 & 6.9 & 0.42 & 7.8 & 5.1 \\
3382_2qPE & 20,23 & 12.9 & 1.13 & 14.3 & 6.8 \\
3468_2hSE & 37,34 & 13.0 & 1.27 & 14.2 & \\
\hline \hline
\end{tabular}




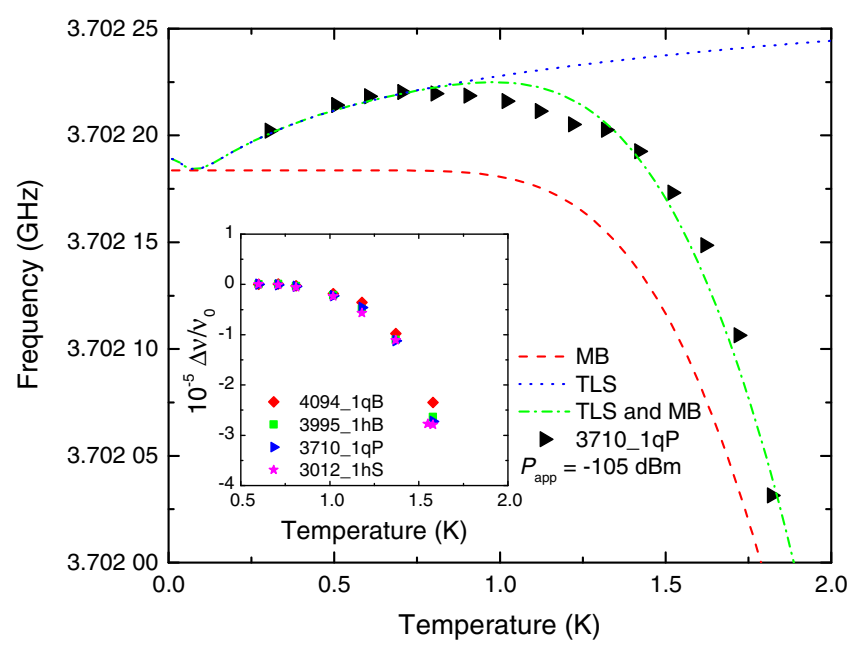

FIG. 2. Resonant frequency of resonator 3710_1_qP as a function of the temperature. Red broken line: Variation arising from kinetic inductance changes described by MB theory. Blue dotted line: Variation arising from TLS losses. Green dash-dotted line: Fit to data including both MB and TLS effects. Inset: Normalized frequency shift as a function of the temperature for all resonators on chip 1 .

the normalized change in resonance frequency, $\alpha$ is the kinetic inductance fraction, and $\sigma_{2}$ is the imaginary part of the complex conductivity as given by Mattis-Bardeen (MB) theory [24]. The inset of Fig. 2 shows the temperature dependence of the resonant frequency for all resonators on chip 1 . The bunching of data points indicates a very similar $T_{c}$ whether the resonator contains nanowires or not, implying that the neon FIB has not significantly suppressed the superconductivity.

Further decreasing the temperature from $1 \mathrm{~K}$, the resonant frequency decreases due to a thermal desaturation of TLSs, which can be described by

$\frac{\nu_{0}(T)-\nu_{0}\left(T_{0}\right)}{\nu_{0}\left(T_{0}\right)}=F \delta_{\mathrm{TLS}}^{i}\left\{\ln \left(\frac{T}{T_{0}}\right)-\left[g(T, \omega)-g\left(T_{0}, \omega\right)\right]\right\}$,

where $g(T, \omega)=\operatorname{Re} \Psi\left(\frac{1}{2}+\hbar \omega / 2 \pi j k_{B} T\right), F$ is the filling factor which typically relates to device geometry and electric field density, $T_{0}$ is a reference temperature, $\Psi$ is the complex digamma function, and $F \delta_{\mathrm{TLS}}^{i}$ is the intrinsic loss tangent. Figure 2 shows a fit to both the MB and TLS frequency shifts, and the extracted $F \delta_{\text {TLS }}^{i}$ is shown in Table I. Barends et al. [25] have previously showed that to determine $F \delta_{\mathrm{TLS}}^{i}$ using both MB and TLS models, it is not necessary to obtain data in the temperature range covering the frequency upturn below $100 \mathrm{mK}$ seen in the TLS fit curve in Fig. 2.

The thermal desaturation of TLS below $1 \mathrm{~K}$ results in absorption of microwave photons, leading to a power- and temperature-dependent resonator loss rate [2,3]. At low microwave drive, the unsaturated TLSs dominate the loss, but as the microwave drive increases, these TLSs become saturated, and, therefore, their loss rate decreases. At high microwave drives, where the TLSs are saturated, the loss becomes dominated by residual quasiparticles, with a loss rate $\delta_{\mathrm{QP}}$ which is temperature dependent but assumed to be independent of microwave power [19]. The TLS and quasiparticle loss behavior can be described by

$$
\frac{1}{Q_{\mathrm{i}}}=\delta_{\mathrm{tot}}^{i}=F \delta_{\mathrm{TLS}}^{0} \frac{\tanh \left(\frac{h \nu_{0}}{2 k_{B} T}\right)}{\left[1+\left(\frac{<n>}{n_{\mathrm{c}}}\right)\right]^{\beta}}+\delta_{\mathrm{QP}},
$$

where $n_{c}$ is the number of photons equivalent to the saturation field of the TLS, $\beta$ describes how quickly the TLSs saturate with power, and $F \delta_{\mathrm{TLS}}^{0}$ is the TLS-loss tangent $\left(F \delta_{\text {TLS }}^{0}\right.$ is power and temperature independent). TLS models were originally based on the anomalous properties of glasses at low temperatures [6] and assumed noninteracting TLSs, which leads to a prediction of $\beta=0.5$. However, as superconducting circuits have improved, this model has failed to accurately describe the power dependence of dielectric losses: a weaker power dependence with $\beta<0.5$ is frequently found [3,26-28]. This shows the need to consider TLS interactions $[4,5,7,26,29]$, changing the loss model to [7,29]

$$
\frac{1}{Q_{i}}=F P_{\gamma} \chi \ln \left(\frac{C n_{c}}{n}+\delta_{\mathrm{QP}}^{\prime}\right) \tanh \left(\frac{h \nu_{0}}{2 k_{B} T}\right),
$$

where $\chi$ is the dimensionless TLS parameter, $P_{\gamma}$ is the TLS switching rate ratio, $C$ is a large constant, and $\delta_{\mathrm{QP}}^{\prime}$ is the log-scaled quasiparticle loss rate.

This loss is examined in more detail by fitting the resonator $S_{21}$ response as a function of microwave drive and temperature [shown in Figs. 3(a)-3(c)]. Figure 3(a) [Fig. 3(c)] shows measurements of $\delta_{\text {tot }}^{i}\left(\right.$ where $\left.\delta_{\text {tot }}^{i}=1 / Q_{i}\right)$ as a function of $\langle n\rangle$ on bare (nanowire-embedded) resonators. Each resonator has its own symbol, with solid (hollow) symbols corresponding to measurements in a normal (ECCOSORB-lined) sample box. ECCOSORB CR-117 (see the Supplemental Material [30]) is a microwave absorber which has been shown to reduce quasiparticle excitation from stray infrared (IR) photons [40]. The ECCOSORB lining is shown in Fig. 1(e). Different colors correspond to different temperatures. When analyzing Figs. 3(a) and 3(c) with Eq. (3), we find $\beta \approx 0.1-0.2$ (see the Supplemental Material [30]) implying interacting TLSs. The solid lines represent fits to the interacting-TLS model, Eq. (4). Table I collects fit parameters from both models.

We first consider bare resonators measured in a standard sample box [solid symbols in Fig. 3(b)]. The resonators on the same chip show a fabrication-based variability also found in the literature $[2,19,41]:$ high- $\langle n\rangle Q_{i}=(1.2-3.1) \times 10^{6}$ and low- $\langle n\rangle Q_{i}=(3.6-5.7) \times 10^{5}$ at $307 \mathrm{mK}$. Increasing 

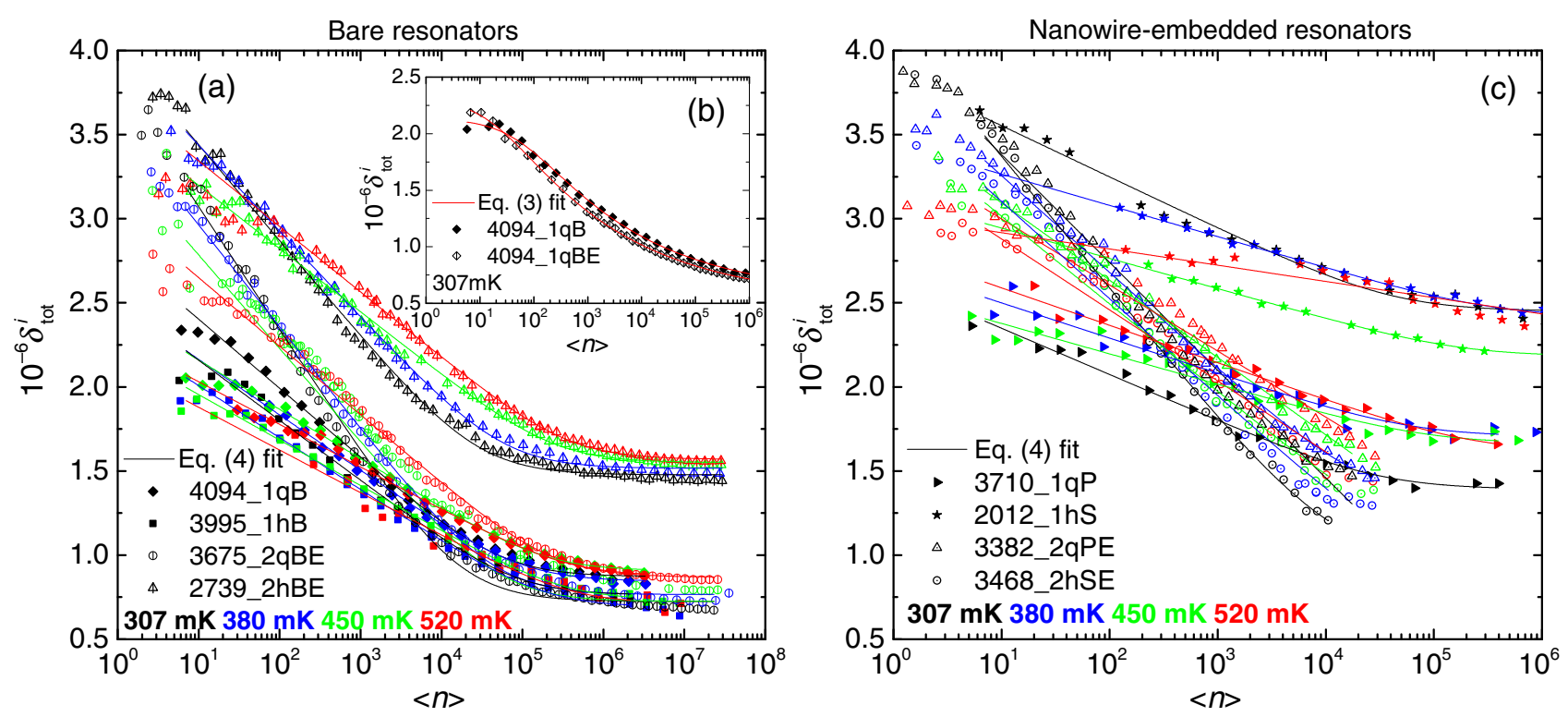

FIG. 3. (a) Loss tangent $\left(\delta_{\mathrm{tot}}^{i}=1 / Q_{i}\right)$ as a function of microwave drive for bare resonators. The colors correspond to different temperatures, and the hollow symbols indicate the use of an ECCOSORB-CR-117-lined sample box. The solid lines in all plots represent fits to Eq. (4). (b) Loss tangent for the resonator 4094_1qB with and without the ECCOSORB-lined box. (c) Loss tangent as a function of microwave drive for the nanowire-embedded resonators.

the temperature leads to an increase in low- $\langle n\rangle Q_{i}$ because, as thermal occupation of the TLSs increases, their ability to absorb microwave photons decreases [3,41], as described by the tanh temperature term. Increasing temperature also leads to a decrease in high- $\langle n\rangle Q_{i}$. This is due to a higher quasiparticle density, meaning that more energy is lost to the quasiparticle system [19].

We next consider nanowire-embedded resonators in the standard sample box [solid symbols in Fig. 3(c)]. At $307 \mathrm{mK}$, at low $\langle n\rangle$, we find $Q_{i}=(2.7-3.9) \times 10^{5}$, in good agreement with the results from the bare resonators, so the FIB-based fabrication of the nanowire has produced very little additional TLS loss. At high $\langle n\rangle$, we find $Q_{i}=(4.1-7.2) \times 10^{5}$, 3-5 times lower than the bare resonators, indicating a higher residual quasiparticle density for the nanowire-embedded resonators.

Quasiparticles generated from pair-breaking events are an important consideration in conventional Josephson-junction devices [42], where ECCOSORB is typically used to reduce quasiparticle-based losses caused by stray IR photons $[40,42]$. We examine whether quasiparticles generated from IR photons are important for nanowire-embedded resonators by measuring them in an ECCOSORB-lined sample box. As the hollow dotted symbols in Fig. 3(c) show, losses at high $\langle n\rangle$ are much lower than for the standard sample box and $Q_{i} \approx(6-9) \times 10^{5}$. This value matches that of the bare resonators for the same $\langle n\rangle$, suggesting that the density of residual quasiparticles is reduced to that of the bare resonators (see Table I). A saturated high- $\langle n\rangle Q_{i}$ is not observed, due to nonlinearities in the resonance line shape of the nanowire-embedded resonators. With the smaller quasiparticle-based loss, the TLS-based low- $\langle n\rangle$ trend of loss increasing as $\langle n\rangle$ decreases is once again found. The high- $\langle n\rangle Q_{i}$ is found to increase with increasing temperature, consistent with losses from thermally generated quasiparticles as found in the bare resonators, indicating that increased quasiparticle losses in nanowireembedded resonators in the normal sample box arise from quasiparticles excited by IR photons. As Table I shows, $\delta_{\mathrm{QP}}$ of the nanowire-embedded resonators in the ECCOSORB environment match those of the bare resonators (both with and without the ECCOSORB environment) and are, therefore, limited by another mechanism which is not unique to the nanowire.

Figure 3(b) shows the loss for the same bare resonator with and without the ECCOSORB enclosure. In contrast to nanowire-embedded resonators, the high- $\langle n\rangle$ loss decreases only slightly when the ECCOSORB-lined sample box is used. This is actually unsurprising since the energy gap of $\mathrm{NbN}$ is approximately 10 times larger than in $\mathrm{Al}$. On the other hand, the reason for the sensitivity to IR photons in the nanowire-embedded resonators is not immediately obvious. Our results demonstrate the importance of IR filtering even when nanowires have a large superconducting energy gap such as those in $\mathrm{NbN}$. This finding is relevant to all nanowire-based devices. We note that a small suppression of $T_{c}$ in our nanowire (below the precision of our $T_{c}$ determination) can give some enhanced sensitivity to IR photons. Alternative explanations for the sensitivity include the nanowire exhibiting a different quasiparticle lifetime [43] or nonequilibrium superconductivity [44], but these are beyond the scope of this study, although, since $Q_{i}$ 
remains high, the number of quasiparticles created from IR photons must still be quite small [40].

Finally, we compare the consistency of the TLS-loss rates (Table I and Supplemental Material [30]) obtained from the analysis of the data shown in Figs. 2 and 3. $F \delta_{\mathrm{TLS}}^{i}$ and $F \delta_{\mathrm{TLS}}^{0}$ differ by less than 20\%; this difference is because $F \delta_{\mathrm{TLS}}^{0}$ is sensitive only to near-resonant TLSs, whereas $F \delta_{\mathrm{TLS}}^{i}$ is also sensitive to a broad spectrum of off-resonant TLSs $[3,41,45]$. Next, we note that $\delta_{\mathrm{TLS}}^{i}=\chi[7]$ so that the ratio $F P_{\gamma} \chi / F \delta_{\mathrm{TLS}}^{i}$ gives $P_{\gamma}$. We find an average value of $P_{\gamma}=0.093$. This agrees well (see the Supplemental Material [30]) with the charge noise spectra of single-electron transistors that give $P_{\gamma} \approx 0.10$ [46,47]. Therefore, all TLS-loss rates are consistent with each other. The TLS-loss rates imply a TLS-limited $Q_{i}$ up to approximately $2 \times 10^{5}$ in the quantum limit (at temperatures down to $10 \mathrm{mK}$ and at single-photon energies). This value is approximately $100 \times$ larger than in equivalent nanowire-embedded resonators and compares favorably with Josephson-junction-embedded resonators.

\section{CONCLUSION}

To conclude, we use a neon FIB to create superconducting nanowires with widths down to $20 \mathrm{~nm}$ within superconducting resonators. In the low-power limit, these devices demonstrate $Q_{i}$ up to $3.9 \times 10^{5}$ at $300 \mathrm{mK}$, with $\delta_{\mathrm{TLS}}^{i}$ and $\delta_{\mathrm{TLS}}^{0}$ corresponding to a TLS-limited $Q_{i}$ up to $2 \times 10^{5}$ at $10 \mathrm{mK}$. These TLS losses arise from the $\mathrm{NbN}$ thin-film technology rather than the neon FIB, meaning a higher $Q_{i}$ should be possible with better resonator technology [41]. By obtaining such a high $Q_{i}$ using nanowires, we demonstrate a critical step towards realizing nanowirebased, superinductance, phase-slip or Dayem-bridge circuits with coherence times comparable to conventional Josephson-junction-type devices.

\section{ACKNOWLEDGMENTS}

The authors acknowledge useful discussions with S. de Graaf, E. Dupont-Ferrier, N. Constantino, and T. Lindström. We also thank T. Lindström for the loan of equipment and critical reading of the manuscript. The authors gratefully acknowledge funding from the United Kingdom Engineering and Physical Sciences Research Council, Grants No. EP/ J017329/1 (J. B. and J. C. F.) and No. EP/K024701/1 (J. S. and P. A. W.), and Carl Zeiss Semiconductor Manufacturing Technology (J. S., O. W. K., and P. A. W.).

[1] P. J. J. O'Malley et al., Scalable Quantum Simulation of Molecular Energies, Phys. Rev. X 6, 031007 (2016).

[2] T. Lindström, J. E. Healey, M.S. Colclough, C. M. Muirhead, and A. Ya. Tzalenchuk, Properties of superconducting planar resonators at millikelvin temperatures, Phys. Rev. B 80, 132501 (2009).
[3] P. Macha, S. H. W. van der Ploeg, G. Oelsner, E. Il'ichev, H.-G. Meyer, S. Wnsch, and M. Siegel, Losses in coplanar waveguide resonators at millikelvin temperatures, Appl. Phys. Lett. 96, 062503 (2010).

[4] J. Burnett, L. Faoro, I. Wisby, V. L. Gurtovoi, A. V. Chernykh, G. M. Mikhailov, V. A. Tulin, R. Shaikhaidarov, V. Antonov, P. J. Meeson, A. Ya. Tzalenchuk, and T. Lindström, Evidence for interacting two-level systems from the $1 / f$ noise of a superconducting resonator, Nat. Commun. 5, 4119 (2014).

[5] A. N. Ramanayaka, B. Sarabi, and K. D. Osborn, Evidence for universal relationship between the measured $1 / f$ permittivity noise and loss tangent created by tunneling atoms, arXiv:1507.06043.

[6] W. A. Phillips, Tunneling states in amorphous solids, J. Low Temp. Phys. 7, 351 (1972).

[7] Lara Faoro and Lev B. Ioffe, Interacting tunneling model for two-level systems in amorphous materials and its predictions for their dephasing and noise in superconducting microresonators, Phys. Rev. B 91, 014201 (2015).

[8] L. Hao, D. C. Cox, and J. C. Gallop, Characteristics of focused ion beam nanoscale Josephson devices, Supercond. Sci. Technol. 22, 064011 (2009).

[9] E. M. Levenson-Falk, R. Vijay, and I. Siddiqi, Nonlinear microwave response of aluminum weak-link Josephson oscillators, Appl. Phys. Lett. 98, 123115 (2011).

[10] O. V. Astafiev, L. B. Ioffe, S. Kafanov, Yu. A. Pashkin, K. Yu. Arutyunov, D. Shahar, O. Cohen, and J. S. Tsai, Coherent quantum phase slip, Nature (London) 484, 355 (2012).

[11] J. T. Peltonen, Z. H. Peng, Yu. P. Korneeva, B. M. Voronov, A. A. Korneev, A. V. Semenov, G. N. Gol'tsman, J. S. Tsai, and O. V. Astafiev, Coherent dynamics and decoherence in a superconducting weak link, Phys. Rev. B 94, 180508 (2016).

[12] Andrey Belkin, Matthew Brenner, Thomas Aref, Jaseung $\mathrm{Ku}$, and Alexey Bezryadin, Little-Parks oscillations at low temperatures: Gigahertz resonator method, Appl. Phys. Lett. 98, 242504 (2011).

[13] C. H. Webster, J. C. Fenton, T. T. Hongisto, S. P. Giblin, A. B. Zorin, and P. A. Warburton, NbSi nanowire quantum phaseslip circuits: dc supercurrent blockade, microwave measurements, and thermal analysis, Phys. Rev. B 87, 144510 (2013).

[14] K. K. Likharev, Superconducting weak links, Rev. Mod. Phys. 51, 101 (1979).

[15] Mark David Jenkins, Uta Naether, Miguel Ciria, Javier Ses, James Atkinson, Carlos Snchez-Azqueta, Enrique del Barco, Johannes Majer, David Zueco, and Fernando Luis, Nanoscale constrictions in superconducting coplanar waveguide resonators, Appl. Phys. Lett. 105, 162601 (2014).

[16] S. E. de Graaf, J. Leppäkangas, A. Adamyan, A. V. Danilov, T. Lindström, M. Fogelström, T. Bauch, G. Johansson, and S. E. Kubatkin, Charge Qubit Coupled to an Intense Microwave Electromagnetic Field in a Superconducting $\mathrm{Nb}$ Device: Evidence for Photon-Assisted Quasiparticle Tunneling, Phys. Rev. Lett. 111, 137002 (2013).

[17] M. Simoen, C. W. S. Chang, P. Krantz, Jonas Bylander, W. Wustmann, V. Shumeiko, P. Delsing, and C. M. Wilson, Characterization of a multimode coplanar waveguide parametric amplifier, J. Appl. Phys. 118, 154501 (2015). 
[18] C. M. Quintana et al., Characterization and reduction of microfabrication-induced decoherence in superconducting quantum circuits, Appl. Phys. Lett. 105, 062601 (2014).

[19] Jan Goetz, Frank Deppe, Max Haeberlein, Friedrich Wulschner, Christoph W. Zollitsch, Sebastian Meier, Michael Fischer, Peter Eder, Edwar Xie, Kirill G. Fedorov, Edwin P. Menzel, Achim Marx, and Rudolf Gross, Loss mechanisms in superconducting thin film microwave resonators, J. Appl. Phys. 119, 015304 (2016).

[20] R. Timilsina, S. Tan, R. Livengood, and P. D. Rack, Monte Carlo simulations of nanoscale focused neon ion beam sputtering of copper: Elucidating resolution limits and sub-surface damage, Nanotechnology 25, 485704 (2014).

[21] F. H. M. Rahman, Shawn McVey, Louis Farkas, John A. Notte, Shida Tan, and Richard H. Livengood, The prospects of a subnanometer focused neon ion beam, Scanning 34, 129 (2012).

[22] T. T. Hongisto and A. B. Zorin, Single-Charge Transistor Based on the Charge-Phase Duality of a Superconducting Nanowire Circuit, Phys. Rev. Lett. 108, 097001 (2012).

[23] S. Probst, F. B. Song, P. A. Bushev, A. V. Ustinov, and M. Weides, Efficient and robust analysis of complex scattering data under noise in microwave resonators, Rev. Sci. Instrum. 86, 024706 (2015).

[24] D. C. Mattis and John Bardeen, Theory of the anomalous skin effect in normal and superconducting metals, Phys. Rev. 111, 412 (1958).

[25] R. Barends, H. L. Hortensius, Tony Zijlstra, Jochem J. A. Baselmans, S. J. C. Yates, J. R. Gao, and Teun M. Klapwijk, Noise in NbTiN, Al, and Ta superconducting resonators on silicon and sapphire substrates, IEEE Trans. Appl. Supercond. 19, 936 (2009).

[26] J. Burnett, L. Faoro, and T. Lindstrm, Analysis of high quality superconducting resonators: consequences for TLS properties in amorphous oxides, Supercond. Sci. Technol. 29, 044008 (2016).

[27] David S. Wisbey, Jiansong Gao, Michael R. Vissers, Fabio C. S. da Silva, Jeffrey S. Kline, Leila Vale, and David P. Pappas, Effect of metal/substrate interfaces on radiofrequency loss in superconducting coplanar waveguides, J. Appl. Phys. 108, 093918 (2010).

[28] M.S. Khalil, F. C. Wellstood, and K. D. Osborn, Loss dependence on geometry and applied power in superconducting coplanar resonators, IEEE Trans. Appl. Supercond. 21, 879 (2011).

[29] Lara Faoro and Lev B. Ioffe, Internal Loss of Superconducting Resonators Induced by Interacting Two-Level Systems, Phys. Rev. Lett. 109, 157005 (2012).

[30] See Supplemental Material at http://link.aps.org/ supplemental/10.1103/PhysRevApplied.8.014039 for details of the experimental setup and further discussion of TLS losses, which includes Refs. [31-39].

[31] D. F. Santavicca and D. E. Prober, Impedance-matched low-pass stripline filters, Meas. Sci. Technol. 19, 087001 (2008).

[32] Simon Gustavsson, Fei Yan, Gianluigi Catelani, Jonas Bylander, Archana Kamal, Jeffrey Birenbaum, David Hover, Danna Rosenberg, Gabriel Samach, Adam P. Sears, Steven J. Weber, Jonilyn L. Yoder, John Clarke, Andrew J.
Kerman, Fumiki Yoshihara, Yasunobu Nakamura, Terry P. Orlando, and William D. Oliver, Suppressing relaxation in superconducting qubits by quasiparticle pumping, Science 354, 1573 (2016).

[33] R. P. Budoyo, J. B. Hertzberg, C. J. Ballard, K. D. Voigt, Z. Kim, J. R. Anderson, C. J. Lobb, and F. C. Wellstood, Effects of nonequilibrium quasiparticles in a thin-film superconducting microwave resonator under optical illumination, Phys. Rev. B 93, 024514 (2016).

[34] Jürgen Lisenfeld, Grigorij J. Grabovskij, Clemens Müller, Jared H. Cole, Georg Weiss, and Alexey V. Ustinov, Observation of directly interacting coherent twolevel systems in an amorphous material, Nat. Commun. 6, 6182 (2015).

[35] S. T. Skacel, Ch. Kaiser, S. Wuensch, H. Rotzinger, A. Lukashenko, M. Jerger, G. Weiss, M. Siegel, and A. V. Ustinov, Probing the density of states of two-level tunneling systems in silicon oxide films using superconducting lumped element resonators, Appl. Phys. Lett. 106, 022603 (2015).

[36] Alexej Semenov, Burghardt Günther, Ute Böttger, H.-W. Hübers, Holger Bartolf, Andreas Engel, Andreas Schilling, Konstantin Ilin, Michael Siegel, R. Schneider, D. Gerthsen, and N.A. Gippius, Optical and transport properties of ultrathin $\mathrm{NbN}$ films and nanostructures, Phys. Rev. B 80, 054510 (2009).

[37] P. C. J. J. Coumou, Ph.D. thesis, Delft University of Technology, 2015.

[38] P. J. De Visser, D. J. Goldie, P. Diener, S. Withington, J. J. A. Baselmans, and T. M. Klapwijk, Evidence of a Nonequilibrium Distribution of Quasiparticles in the Microwave Response of a Superconducting Aluminum Resonator, Phys. Rev. Lett. 112, 047004 (2014).

[39] A. A. Adamyan, S. E. Kubatkin, and A. V. Danilov, Tunable superconducting microstrip resonators, Appl. Phys. Lett. 108, 172601 (2016).

[40] R. Barends, J. Wenner, M. Lenander, Y. Chen, R. C. Bialczak, J. Kelly, E. Lucero, P. O’Malley, M. Mariantoni, D. Sank, H. Wang, T. C. White, Y. Yin, J. Zhao, A. N. Cleland, John M. Martinis, and J. J. A. Baselmans, Minimizing quasiparticle generation from stray infrared light in superconducting quantum circuits, Appl. Phys. Lett. 99, 113507 (2011).

[41] A. Bruno, G. de Lange, S. Asaad, K. L. van der Enden, N. K. Langford, and L. DiCarlo, Reducing intrinsic loss in superconducting resonators by surface treatment and deep etching of silicon substrates, Appl. Phys. Lett. 106, 182601 (2015).

[42] Antonio D. Córcoles, Jerry M. Chow, Jay M. Gambetta, Chad Rigetti, J. R. Rozen, George A. Keefe, Mary Beth Rothwell, Mark B. Ketchen, and M. Steffen, Protecting superconducting qubits from radiation, Appl. Phys. Lett. 99, 181906 (2011).

[43] P. J. de Visser, J. J. A. Baselmans, P. Diener, S. J. C. Yates, A. Endo, and T. M. Klapwijk, Number Fluctuations of Sparse Quasiparticles in a Superconductor, Phys. Rev. Lett. 106, 167004 (2011).

[44] D. J. Goldie and S. Withington, Non-equilibrium superconductivity in quantum-sensing superconducting resonators, Supercond. Sci. Technol. 26, 015004 (2013). 
[45] D. P. Pappas, M. R. Vissers, D. S. Wisbey, J. S. Kline, and J. Gao, Two level system loss in superconducting microwave resonators, IEEE Trans. Appl. Supercond. 21, 871 (2011).

[46] S. Kafanov, H. Brenning, T. Duty, and P. Delsing, Charge noise in single-electron transistors and charge qubits may be caused by metallic grains, Phys. Rev. B 78, 125411 (2008).

[47] This value arises from $P_{\gamma}=1 / \ln \left(\gamma_{\max } / \gamma_{\min }\right)$, where $\gamma_{\max }$ $\left(\gamma_{\min }\right)$ is the maximum (minimum) rate of TLS switching. For a TLS charge noise spectrum extending from a few hertz to a few $100 \mathrm{kHz}, P_{\gamma} \approx 0.1$. 